Educación Física y Ciencia, vol. 19, n² 2, e039, diciembre 2017. ISSN 2314-2561

Universidad Nacional de La Plata.

Facultad de Humanidades y Ciencias de la Educación.

Departamento de Educación Física

\title{
Estilo de vida de estudantes de Educação Física
}

\author{
Life style of Physical Education Students
}

\author{
João Miguel de Souza Neto *; Sanderson Soares da Silva **; \\ Michelle Salles de Oliveira $* * *$
}

* Grupo de Estudos e Pesquisas em Epidemiologia da Atividade Física - GEPEAF. Programa Associado de Pós-Graduação em Educação Física - Universidade de Pernambuco/Uiversidade Federal de Paraíba. João Pessoa, PB, Brasil; ** Universidade Federal da Paraíba - UFPB. Grupo de Estudos e Pesquisas em Epidemiologia da Atividade Física - GEPEAF. João Pessoa, PB, Brasil; *** Centro Universitário de João Pessoa UNIPÊ. João Pessoa, PB, Brasil. Programa de Pós-Graduação em Ciência da Terra - Universidade Fernando Pessoa, Portugal I miguel.edf@hotmail.com; ssparesdasilva@sinmail.com; msallesdeoliveira@sinmail.com

\section{PALAVRAS-CHAVE}

Estudante Universitário

Estilo de vida

Atividade física

\section{KEYWORDS}

University Student

Life Style

Physical Activity

\section{RESUMO}

O objetivo do presente estudo foi descrever o estilo de vida e a atividade física habitual em universitários de Educação Física de uma Instituição privada de Ensino Superior. Este é um estudo descritivo, com delineamento transversal. A amostra foi composta por 82 universitários, de ambos os sexos, do curso de Educação Física de uma instituição de Ensino Superior, João Pessoa, Paraíba, Brasil. Foram utilizados dois questionários: Perfil do Estilo de Vida; Questionário de Atividades Físicas Habituais. Os dados coletados foram tratados por meio da estatística descritiva (média e desvio padrão, distribuição por frequência) no software SPSS versão 17.0. Os resultados encontrados revelaram que os níveis de estilo de vida dos universitários, apresentam pelo menos um escore negativo em cada um dos cinco componentes estudados, ficou evidenciado que os componentes nutrição e estresse apresentam os maiores índices de escores negativos. Quanto a análise do nível de atividade física habitual, os dados apresentam-se como bons indicadores do nível de vida fisicamente ativo da população do estudo. No entanto, uma parcela dos participantes apresentou índice indesejável, consequentemente, baixos níveis para a saúde geral. Para concluir, as variáveis hábitos alimentares, prevenção e relacionamento interpessoal necessitam maior atenção dos profissionais de saúde.

\section{ABSTRACT}

The main purpose of this is study was to describe the life style and physical activity habits of the Physical Education students from a private Institution of higher Education. With a cross-sectional design study, the sample was formed by 82 students, both genders, recruited in a Physical Education Course, from a private institution of Higher Education, in João Pessoa, Paraíba, Brazil. To collect data, the students answered two questionnaires: The profile of life styles and the Habitual Physical Activity Questionnaire. The data are presented by descriptive statistics, (means, standard deviation and frequencies) and analyzed in SPSS 17.0. The main results showed at least one negative score in each of the five dimensions of the profile of life styles. In this context, stress and nutrition presented higher negative scores. According the Habitual Physical activity, the data showed good scores in physical activity for this sample. However, a significant number of students presented low levels for this variable, consequently, higher levels for bad health status. To conclude, the variable nutrition habits, prevention and interpersonal relationship need more attention from health professionals. 


\section{Introdução}

Nos dias atuais, devido ao estilo de vida contemporâneo, constata-se que alguns maus hábitos no dia-a-dia das pessoas têm impacto direto na saúde da população e aumentando, consequentemente, a prevalência de baixa qualidade de vida. Isso tem ocorrido a pessoas de todas as idades e condições sociais (Hallal et al., 2012).

Diante deste panorama, o estilo de vida vem, em sua gênese, passando a ser um dos determinantes mais preciosos para a saúde da população (Bauman et. al., 2012). Baseado nisto, é de extrema importância a busca de um estilo de vida ativo, a fim de minimizar problemas centrais, determinantes de uma baixa qualidade de vida que, possivelmente, levaria a um maior risco à saúde.

Assim, quando falamos em estilo de vida, podemos recair no discurso comum, devido à complexidade e imprecisão associada à definição de um conceito de estilo de vida. Para Nahas (2010), o estilo de vida representa o “conjunto de ações habituais que refletem as atitudes, os valores e as oportunidades na vida das pessoas”. No que concerne ao modelo ativo de estilo de vida, podemos entender uma relação inversamente proporcional entre a inatividade física e saúde, considerando aquela uma consequência dos novos hábitos da sociedade moderna. Isto é, a ausência de atividade física é um fator de risco.

Desta forma, uma alternativa para uma vida saudável é a sistematização da prática da atividade física regular, pois esta tem proporcionado benefícios como reduzir a incidência das doenças crônico-degenerativas, como constatado em alguns estudos (Cruz, 2006). Entretanto, um estudo epidemiológico demonstrou que alguns estilos de vida favorecem o desencadeamento do alto índice de inatividade física em países devolvidos e/ou em desenvolvimento, sendo responsável por mais de 3 milhões de mortes por ano (Pratt et al., 2012).

Dados da Organização Mundial da Saúde (OMS, 2006), sugerem que o estilo de vida é um indicador associado com características pessoais que se diferenciam em função das condições em que vive cada ser humano. Todavia, indivíduos com acesso direto às informações sobre um estilo de vida saudável tendem, na prática, a não adotar um estilo de vida mais ativo. Diante dessa realidade, mais do que nunca, é de suma importância a realização de que venham identificar diferentes estilos de vida, identificando aqueles indivíduos mais ativos, identificando um possível processo de revigoramento da saúde e, assim, promover uma melhor qualidade de vida através da prática de hábitos saudáveis (Coelho \& Santos, 2006).

Desta forma, faz-se necessário o levantamento de informações sobre os domínios ligados ao estilo de vida dos universitários da área da saúde, profissionais ligados tanto à prevenção quanto a promoção e tratamento em saúde. Além disso, considerando a áreas de formação e atuação em Educação Física (bacharel e licenciatura), um maior conhecimento nessa área, pode ter grandes implicações em saúde pública, bem como poderá subsidiar o planejamento e o desenvolvimento de programas de intervenção. Sendo assim, o presente estudo tem como objetivo descrever o estilo de vida e a atividade física habitual em universitários de Educação Física de uma instituição particular de ensino superior de João Pessoa - PB, Brasil.

\section{Metodologia}

Trata-se de um estudo descritivo, com delineamento transversal. A amostra foi constituída por 98 universitários de ambos os sexos, matriculados no curso de Educação Física, Licenciatura $(n=44)$ e Bacharelado $(n=54)$, do Centro Universitário de João Pessoa - UNIPÊ, João Pessoa, Paraíba, Brasil.

A coleta de dados ocorreu entre março e abril de 2012, sendo realizada por equipe treinada em um estudo piloto. O questionário foi preenchido de próprio punho, em sala de aula, no horário regular, seguindo as instruções prévias, fornecidas pela equipe de coleta de dados. Para reduzir o número de perdas, o questionário foi aplicado de terça a quinta-feira e no segundo horário de aula, horário este que conta com maior número de alunos presentes.

Os critérios de inclusão foram: estar devidamente matriculado no curso de Educação Física no primeiro semestre letivo do ano de 2012 e aceitarem voluntariamente participar do estudo. Foram excluídos da pesquisa os indivíduos que apresentaram alguma condição física, psicológica ou cognitiva que impossibilitasse de responder 
ao questionário.

As variáveis sociodemográficas foram coletadas por meio de questões pré-codificadas que abrangiam (sexo, idade, escolaridade materna, estado civil, curso, cor da pele e classe econômico - CE). Ainda, foram coletados dados sobre a classe econômica (critérios sugeridos pela Associação Brasileira de Empresas de Pesquisa - ABEP), que leva em consideração a presença de bens materiais e o número de empregados mensalistas na residência, a escolaridade do chefe da família, agrupando as pessoas nas seguintes classes econômicas: A1, A2 [alta], B1, B2, C1, C2, D e E [baixa].

Com base nos objetivos da pesquisa, optou-se pela utilização do instrumento constituído de um questionário denominado de Perfil do Estilo de Vida, proposto por Nahas, Barros e Francalacci (2000), que quantifica o nível de estilo de vida dos universitários nos aspectos nutrição, atividade física, comportamento preventivo, relacionamentos e estresse. Esse questionário é constituído por quinze questões, classificando o nível de estilo de vida em: 0 e 1 para o perfil negativo; 2 e 3 para perfil positivo, correspondente às afirmações das três opções existentes nos cinco componentes

Nesses cinco domínios investigados, foi feito um demonstrativo gráfico representado pelo Pentáculo do Bemestar, referente ao estilo de vida da população do estudo consubstanciado pelas respostas dos 15 itens do questionário. Desta forma, preenchemos a Figura 2 e 3 do Pentáculo, em que os espaços em branco significam que o grupo marcou zero; do centro até o primeiro círculo, que marcou 1; do centro até o segundo círculo, que marcou 2; e do centro até o terceiro círculo, que marcou 3. Todas estas afirmações quantitativas foram feitas através das médias aritméticas de cada componente.

O nível de atividade física foi avaliado através do Questionário de Atividades Físico-Habitual proposto por Nahas (2010). Este instrumento é composto por três questões, sendo uma delas com três alternativas, com perguntas referentes a atividades físicas ocupacionais; e oito questões, contendo as duas últimas com três alternativas cada, contendo questões referentes a atividades físicas de lazer. A classificação é feita através das seguintes categorias: 0-5 pontos, inativo; 6-11 pontos, pouco ativo; $12-20$ pontos, moderadamente ativo; e acima de 20 pontos, muito ativo.

Os dados foram analisados no programa Statistical Package for Social Science - IBM, versão SPSS 17.0. Foi utilizada a estatística descritiva, composta por médias e desvio padrão, para a caracterização dos dados numéricos e categóricos através do estudo das medidas de tendência central e de variação. Fez-se também uso de gráficos de setores e histogramas de frequências simples e percentuais para os valores obtidos na pesquisa. O projeto foi aprovado pelo Comitê de Ética do Centro Universitário de João Pessoa - UNIPÊ, (Protocolo 045/11, em 20/12/2011). Os sujeitos foram informados sobre os objetivos do estudo e foi solicitada a assinatura do Termo de Consentimento Livre e Esclarecido

\section{Resultados}

A amostra final foi constituída por 91 estudantes universitários. Houve, ainda, três recusas $(n=3)$ e perdas $(n=4$; não foram encontrados após três visitas de retorno para a realização da pesquisa), totalizando 6,4 \% do total de alunos. Ao fim, a amostra final considerada para este estudo foi de 82 universitários do curso de Educação Física de uma instituição privada, com 26,7 $( \pm 4,6)$ anos de idade, sendo 44 do curso de licenciatura $(27,7 \pm 5,7$ anos de idade) e 38 do bacharelado (22,6 $\pm 3,6$ anos de idade), 65 do sexo masculino e 17 do feminino.

Na Tabela 1, verifica-se as características sociodemográficas dos voluntários (licenciatura e bacharelado). A maioria dos estudantes investigados era do sexo masculino (79,3\%), com até 20 anos de idade (54,9\%), sem companheiro (89,0\%), com escolaridade materna maior que nove anos (58,9\%), de nível econômico alto (74,3\%), e que trabalhavam de forma remunerada $(76,8 \%)$. 
Tabela 1. Características sociodemográficas dos universitários de Educação Física (licenciatura e bacharelado) do município João Pessoa, PB, 2012.

\begin{tabular}{|c|c|c|c|c|c|c|}
\hline \multirow[t]{2}{*}{ Variáveis } & \multicolumn{2}{|c|}{ Bacharelado } & \multicolumn{2}{|c|}{ Licenciatura } & \multicolumn{2}{|c|}{ Todos } \\
\hline & $\mathbf{n}$ & $\%$ & $\mathbf{n}$ & $\%$ & $\mathbf{n}$ & $\%$ \\
\hline \multicolumn{7}{|l|}{ Sexo } \\
\hline Masculino & 30 & 36,6 & 35 & 42,7 & 65 & 79,3 \\
\hline Feminino & 8 & 9,7 & 9 & 11,0 & 17 & 20,7 \\
\hline \multicolumn{7}{|l|}{ Idade (anos) } \\
\hline$\leq 20$ anos & 25 & 30,5 & 20 & 24,4 & 45 & 54,9 \\
\hline$>20$ anos & 15 & 18,3 & 22 & 26,8 & 37 & 45,1 \\
\hline \multicolumn{7}{|l|}{ Situação conjugal } \\
\hline Sem companheiro & 35 & 42,7 & 38 & 46,3 & 73 & 89,0 \\
\hline Com companheiro & 4 & 4,9 & 5 & 6,1 & 9 & 11,0 \\
\hline \multicolumn{7}{|c|}{ Escolaridade da mãe } \\
\hline$\leq 8$ anos & 18 & 22,0 & 16 & 19,5 & 34 & 41,5 \\
\hline$>9$ anos & 25 & 30,5 & 23 & 28,0 & 48 & 58,5 \\
\hline \multicolumn{7}{|l|}{ Classe econômica } \\
\hline Alta & 26 & 31,7 & 35 & 42,7 & 61 & 74,3 \\
\hline Baixa & 10 & 12,2 & 11 & 13,4 & 21 & 25,6 \\
\hline \multicolumn{7}{|c|}{ Trabalho Remunerado } \\
\hline Sim & 37 & 45,1 & 26 & 31,7 & 76,8 & 76,8 \\
\hline Não & 8 & 9,8 & 11 & 13,4 & 23,2 & 23,2 \\
\hline
\end{tabular}

A seguir, apresenta-se os respectivos domínios, dentro de uma sistematização por todos os componentes avaliados, identificando que os dados dos escores são atribuídos através de uma média por cada elemento, numa escala de que vai de (0) zero a (3) três. Os itens que apresentam médias acima de dois (2), consideram-se positivo; abaixo desse valor, a média foi considerada negativa, ou seja, os escores de médias (0) ou (1) representam um perfil de estilo de vida negativo, e os que contêm médias (2) e (3) são considerados positivos, com um estilo de vida saudável.

Tabela 2 - Distribuição dos valores da média e desvio padrão dos cincos componentes do estilo de vida do curso de Bacharelado do município João Pessoa, PB, 2012.

\begin{tabular}{|l|l|}
\hline DESCRIÇÃO DOS PERFIS DO ESTILO DE VIDA & $\mathbf{X} \pm \mathbf{D P}$ \\
\hline COMPONENTE: NUTRIÇÃO & $1,1 \pm 0,9$ \\
\hline A. Sua alimentação diária inclui ao menos 05 (cinco) porções de frutas e verduras? & $1,5 \pm 1,0$ \\
\hline B. Você evita ingerir alimentos gordurosos (carnes gordas, frituras) e doces? & $1,7 \pm 1,2$ \\
\hline C. Você faz 04 (quatro) a 05 (cinco) refeições variadas ao dia, incluindo café da manhã completo? & $\mathbf{X} \pm \mathbf{D P}$ \\
\hline COMPONENTE: ATIVIDADE FÍSICA & $2,2 \pm 0,9$ \\
\hline $\begin{array}{l}\text { D. Você realiza ao menos } 30 \text { minutos de atividades físicas moderadas ou intensas, de forma } \\
\text { contínua ou acumulada, 05 ou mais dias na semana? }\end{array}$ & \\
\hline
\end{tabular}




\begin{tabular}{|l|l|}
\hline $\begin{array}{l}\text { E.Ao menos } 02 \text { (duas) vezes por semana você realiza exercícios que envolvam força e } \\
\text { alongamento muscular? }\end{array}$ & $2,2 \pm 0,9$ \\
\hline $\begin{array}{l}\text { F. No seu dia a dia, você caminha ou pedala como meio de transporte e, preferencialmente, quando } \\
\text { tem oportunidade, usa as escadas ao invés do elevador? }\end{array}$ & $1,4 \pm 1,0$ \\
\hline COMPONENTE: COMPORTAMENTO PREVENTIVO & $\mathbf{X} \pm \mathbf{D P}$ \\
\hline G. Você conhece sua pressão arterial, seus níveis de colesterol e procura controlá-los? & $1,5 \pm 1,2$ \\
\hline H. Você não fuma e ingere álcool com moderação menos de 02 (duas) doses ao dia? & $2,1 \pm 1,1$ \\
\hline $\begin{array}{l}\text { I. Você respeita as normas de trânsito (como pedestre, ciclista ou motorista); se dirige, usa sempre } \\
\text { o cinto de segurança e nunca ingere álcool? }\end{array}$ & $2,6 \pm 0,7$ \\
\hline COMPONENTE: RELACIONAMENTO & $\mathbf{X} \pm \mathbf{D P}$ \\
\hline J. Você procura cultivar amigos, e está satisfeito com seus relacionamentos? & $2,6 \pm 0,7$ \\
\hline $\begin{array}{l}\text { K. Seu lazer inclui reuniões com amigos, atividades esportivas em grupo e participação em } \\
\text { associações ou entidades sociais? }\end{array}$ & $2,6 \pm 0,7$ \\
\hline L. Você procura ser ativo em sua comunidade, sentindo-se útil no seu ambiente social? & $1,8 \pm 1,1$ \\
\hline COMPONENTE: CONTROLE DO STRESS & $\mathbf{X} \pm \mathbf{D P}$ \\
\hline M. Você reserva tempo ao menos 05 (cinco) minutos todos os dias para relaxar? & $2,3 \pm 0,9$ \\
\hline N. Você mantém uma discussão sem alterar-se, mesmo quando contrariado? & $1,3 \pm 0,9$ \\
\hline O. Você equilibra o tempo dedicado ao trabalho com o tempo dedicado ao lazer? & $1,8 \pm 0,9$ \\
\hline
\end{tabular}

Legenda: $\mathrm{X}=$ Média amostral / DP = Desvio padrão

Observa-se, com relação ao estilo de vida dos alunos do bacharelado, que a população analisada apresentou os seguintes resultados: perfil negativo no tocante aos componentes nutricionais A, B e C; quando analisamos o item atividade física, apontaram-se escores negativos no item $\mathrm{F}$; na averiguação do componente comportamento preventivo, mostraram-se escores negativos na questão G; já na verificação do aspecto relacionamento interpessoal, os universitários investigados mostraram média negativa no item L; ainda na análise do controle do estresse, constataram-se escores negativos nas questões N e O ( Tabela 2).

A Figura 1 abaixo é a representação do perfil do estilo de vida de todos os universitários de Educação Física do curso de Bacharelado, derivado do modelo do Pentáculo do Bem-estar. 
Figura 1: Pentáculo do Bem-estar do curso de Bacharelado em Educação Física do município João Pessoa, PB, 2012.

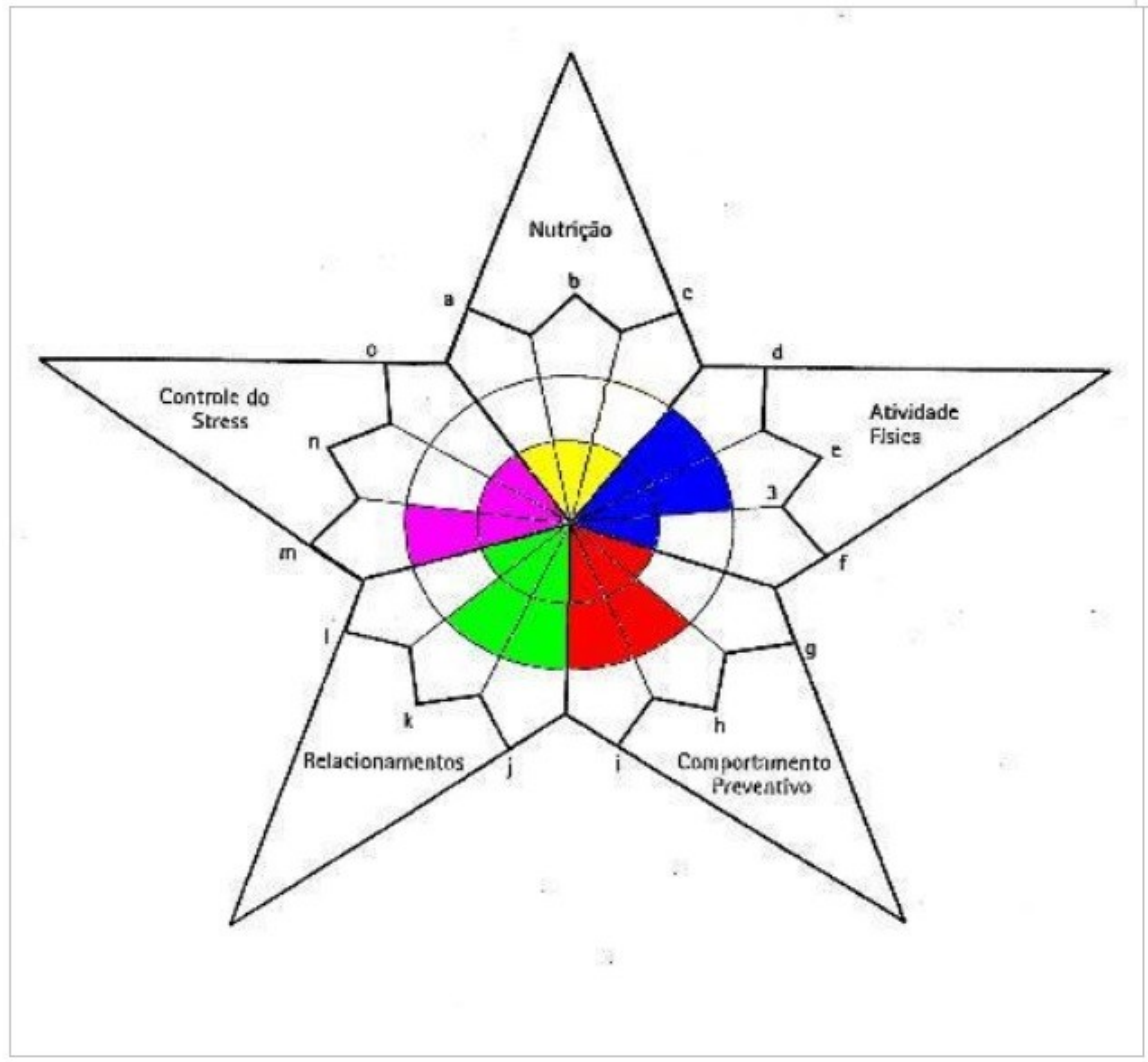

Tabela 3 - Distribuição dos valores da média e desvio padrão dos cincos componentes do estilo de vida do curso de Licenciatura do município João Pessoa, PB, 2012.

\begin{tabular}{|c|c|}
\hline \multicolumn{2}{|l|}{ DESCRIÇÃO DOS PERFIS DO ESTILO DE VIDA } \\
\hline COMPONENTE: NUTRIÇÃO & $\mathbf{X} \pm \mathbf{D P}$ \\
\hline A. Sua alimentação diária inclui ao menos 05 (cinco) porções de frutas e verduras? & $0,9 \pm 0,8$ \\
\hline B. Você evita ingerir alimentos gordurosos (carnes gordas, frituras) e doces? & $1,2 \pm 1,0$ \\
\hline C. Você faz 04 (quatro) a 05 (cinco) refeições variadas ao dia, incluindo café da manhã completo? & $2,2 \pm 0,8$ \\
\hline COMPONENTE: ATIVIDADE FÍSICA & $\mathrm{X} \pm \mathrm{DP}$ \\
\hline $\begin{array}{l}\text { D. Você realiza ao menos } 30 \text { minutos de atividades físicas moderadas ou intensas, de forma } \\
\text { contínua ou acumulada, } 05 \text { ou mais dias na semana? }\end{array}$ & $2,4 \pm 0,8$ \\
\hline $\begin{array}{l}\text { E. Ao menos } 02 \text { (duas) vezes por semana você realiza exercícios que envolvam força e } \\
\text { alongamento muscular? }\end{array}$ & $2,5 \pm 0,7$ \\
\hline $\begin{array}{l}\text { F. No seu dia a dia, você caminha ou pedala como meio de transporte e, preferencialmente, quando } \\
\text { tem oportunidade, usa as escadas ao invés do elevador? }\end{array}$ & $1,6 \pm 1,0$ \\
\hline COMPONENTE: COMPORTAMENTO PREVENTIVO & $\mathrm{X} \pm \mathrm{DP}$ \\
\hline G. Você conhece sua pressão arterial, seus níveis de colesterol e procura controlá-los? & $1,4 \pm 1,1$ \\
\hline H. Você não fuma e ingere álcool com moderação menos de 02 (duas) doses ao dia? & $1,9 \pm 1,1$ \\
\hline $\begin{array}{l}\text { I. Você respeita as normas de trânsito (como pedestre, ciclista ou motorista); se dirige, usa sempre } \\
\text { o cinto de segurança e nunca ingere álcool? }\end{array}$ & $2,3 \pm 0,8$ \\
\hline
\end{tabular}




\begin{tabular}{|l|l|}
\hline COMPONENTE: RELACIONAMENTO & $\mathbf{X} \pm \mathbf{D P}$ \\
\hline J. Você procura cultivar amigos, e está satisfeito com seus relacionamentos? & $2,7 \pm 0,5$ \\
\hline $\begin{array}{l}\text { K. Seu lazer inclui reuniões com amigos, atividades esportivas em grupo e participação em } \\
\text { associações ou entidades sociais? }\end{array}$ & $2,5 \pm 0,7$ \\
\hline L. Você procura ser ativo em sua comunidade, sentindo-se útil no seu ambiente social? & $2,0 \pm 1,0$ \\
\hline COMPONENTE: CONTROLE DO STRESS & $\mathbf{X} \pm \mathbf{D P}$ \\
\hline M. Você reserva tempo ao menos 05 (cinco) minutos todos os dias para relaxar? & $2,3 \pm 0,9$ \\
\hline N. Você mantém uma discussão sem alterar-se, mesmo quando contrariado? & $1,5 \pm 1,1$ \\
\hline O. Você equilibra o tempo dedicado ao trabalho com o tempo dedicado ao lazer? & $1,7 \pm 0,9$ \\
\hline
\end{tabular}

Com relação às respostas dos universitários do curso de licenciatura em Educação Física, percebemos que alguns componentes correspondem aos hábitos nutricionais do licenciado. Ainda, constatamos escores negativos nos itens $\mathrm{A}$ e $\mathrm{B}$; quanto aos aspectos relacionados à atividade física, verificamos escore negativo no item $\mathrm{F}$ quanto às análises feitas dos componentes preventivos; identificamos as características negativas nos itens $\mathrm{G}$ e $\mathrm{H}$; no que se refere ao relacionamento interpessoal, o estudo apresentou média positiva em todos os itens; na investigação do controle do estresse, os resultados apresentam dois itens negativos na questão N e O (Tabela 3).

A Figura 2, abaixo, é a representação do perfil do estilo de vida de todos os universitários do curso de Licenciatura em Educação Física, derivado do modelo do Pentáculo do Bem-estar.

Figura 2: Pentáculo do Bem-estar do curso de Licenciatura em Educação Física do município João Pessoa, PB, 2012.

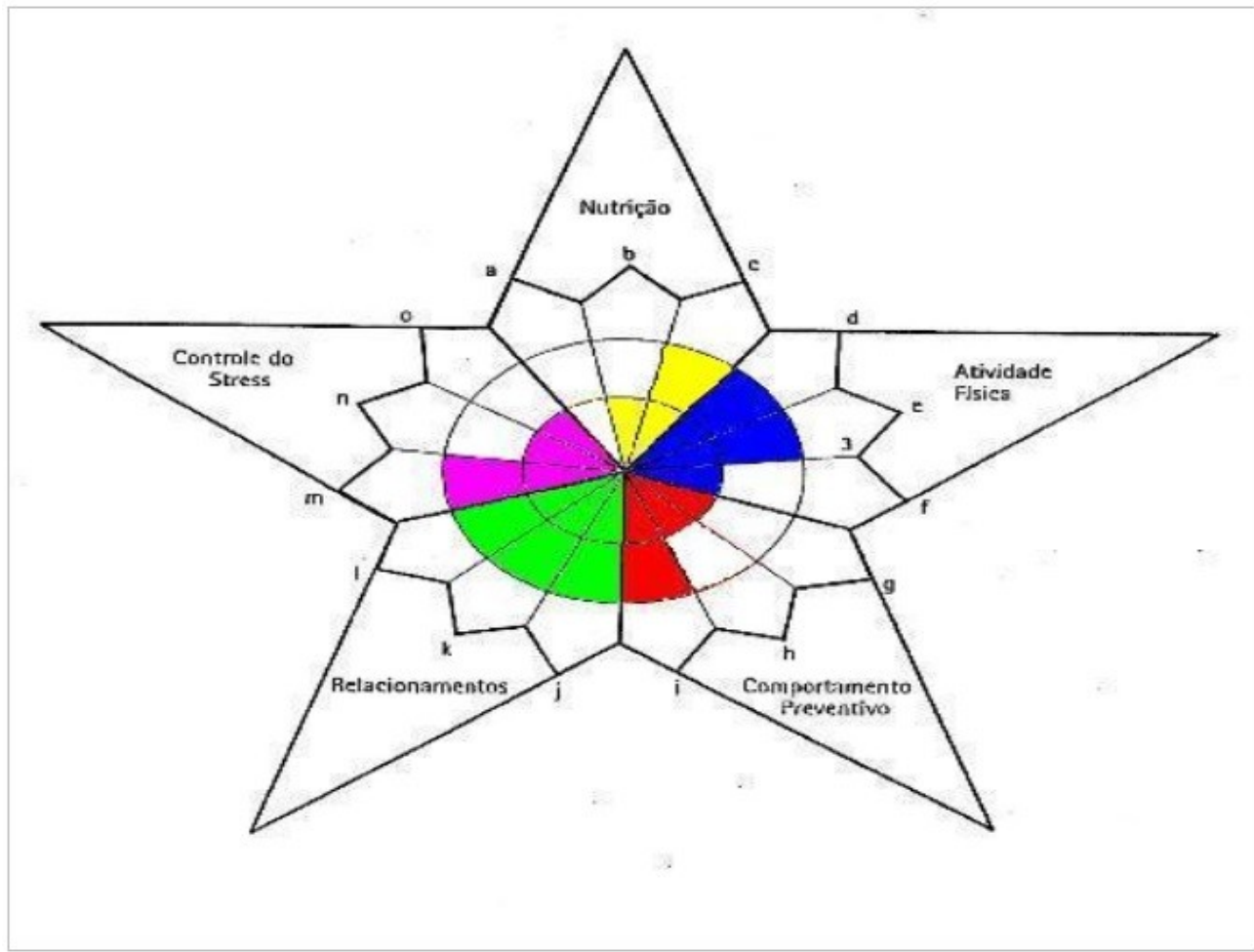

Sobre os indicadores do nível de atividade física habitual, que se divide em quatro categorias (inativo, pouco ativo, moderadamente ativo e muito ativo), as atividades foram classificadas em ocupacionais, de lazer e da vida diária. 
Os resultados apontam, quanto ao indicativo relacionado à saúde, que $71 \%$ dos universitários do curso de Bacharelado em Educação Física são fisicamente ativos. No entanto, 28,9\% dos indivíduos apresentaram baixos índices de atividade física, isso implica dizer que essa parcela de sujeitos não apresenta padrão satisfatório para uma vida fisicamente ativa.

Gráfico - 1 Classificação do nível de atividades físicas habituais dos universitários de Bacharelado em Educação Física do município João Pessoa, PB, 2012.

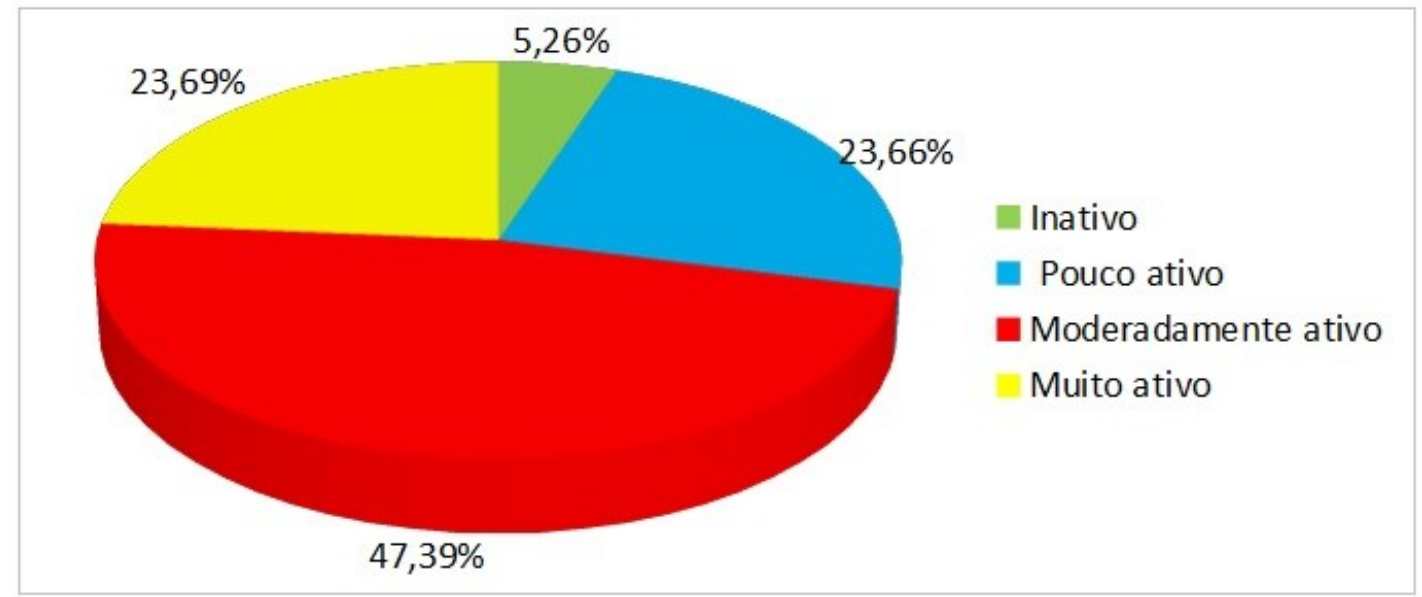

Portanto, os dados obtidos da análise feita sobre os universitários do curso de Licenciatura em Educação Física revelam características inerentes ao grupo considerado fisicamente ativo. Quanto aos fatores de investigação, os aspectos pouco ativo e inativo, não houve percentual para frequência de análise, sendo um dado positivo.

Gráfico - 2 Classificação do nível de atividades físicas habituais dos universitários de Licenciatura em Educação Física do município João Pessoa, PB, 2012.

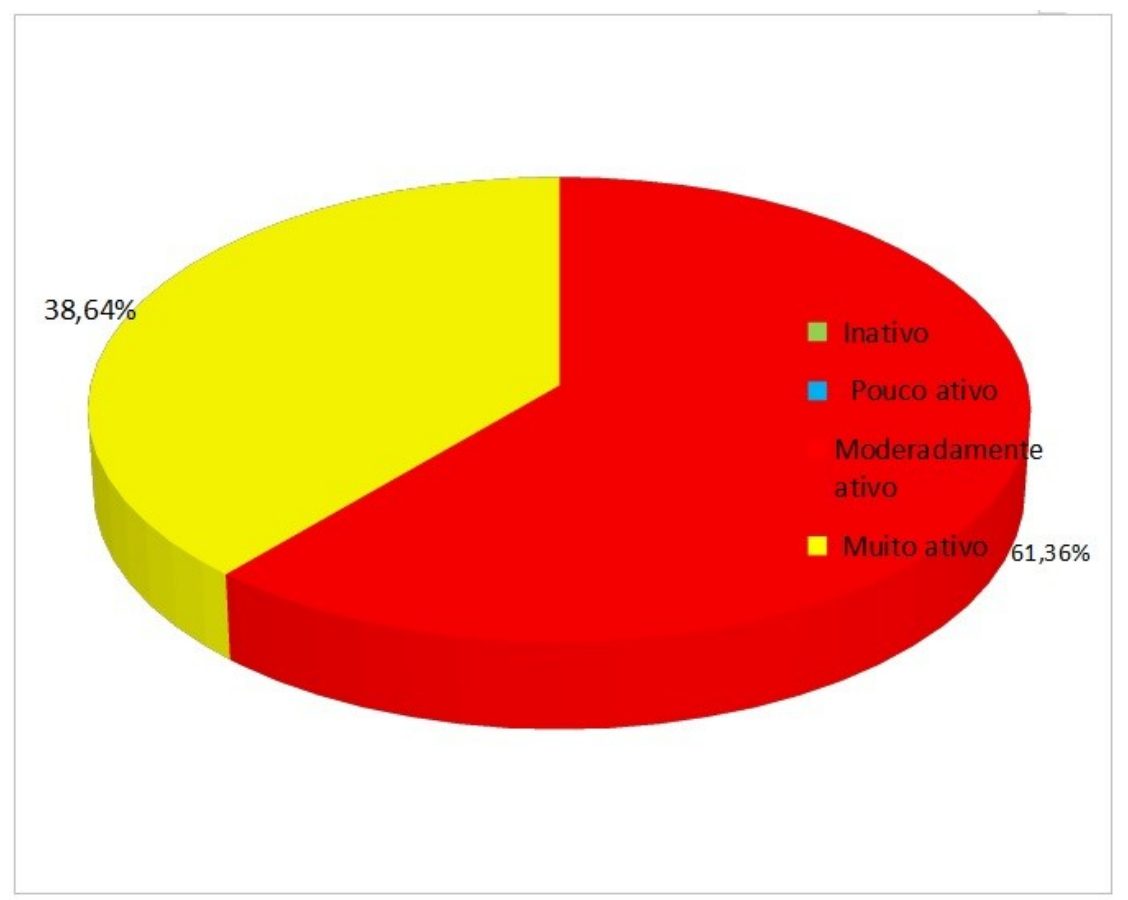




\section{Discussão}

A saúde dos universitários tem sido uma área bastante investigada através do pentáculo do bem-estar, devido à proporção de sua magnitude, por meio de ações de incentivo à aquisição de comportamentos saudáveis (Barboza, Pontes, Feitosa, Nunes \& Sena, 2010; Nogueira, Rossato, Soares, Bezerra \& Moraes, 2015; Santos \& Alves, 2009).

Em geral, os dados encontrados neste estudo estão em conformidade com os realizados por Barboza e colaboradores (2010). Em ambos os estudos, a nutrição apresenta um índice indesejável, revelando que alguns universitários, além de não terem o hábito de se alimentar com frutas ou hortaliças, ingerem alimentos inadequados como carnes gordurosas, frituras e doces nas suas refeições diárias. No que se refere ao componente da atividade física, os alunos realizam práticas corporais que envolvem exercícios físicos regulares com intensidades moderadas e intensas; porém, em relação às atividades ocupacionais diárias, eles manifestam índices negativos.

No componente preventivo, os resultados são próximos ao estudo referenciado, mostrando como fatores negativos itens já identificados, ou seja, o desconhecimento de suas limitações fisiológicas sobre a pressão arterial e o próprio colesterol, além de fumar e ingerir bebida alcoólica. No quesito relacionamento, os universitários têm escores negativos tanto dentro de suas atividades comunitárias, como dentro do âmbito natural e social. E, por fim, outra constante que foi observada com frequência negativa foi a relacionada ao controle do estresse, uma vez que ficou evidenciado que alguns universitários não conseguiram manter-se inalterados durante uma discussão. Outro dado relevante diz respeito a indicação de que não há um padrão diário no equilíbrio entre o tempo de lazer e de trabalho.

Diante do exposto, pode-se constatar que os componentes de maior ocorrência negativa quanto ao estilo de vida foram a "alimentação" e o "controle do estresse", fato relevante e de acordo com os resultados de outras pesquisas brasileiras (Both, Nascimento, Sonoo \& Borgato, 2010). Em outro estudo, Santos e Alves (2009) realizaram um estudo semelhante, com acadêmicos do Sudeste do Brasil. Os autores identificaram que cerca de $79 \%$ dos estudantes apresentavam consumo inadequado de frutas e verduras. Entre os acadêmicos, constatou-se que cerca de $71 \%$ deles não evitavam comer frituras e doces no seu cotidiano (Santos \& Alves, 2009). Essa falta de alimentação adequada nos universitários é atribuída ao fator "tempo restrito" para se alimentar corretamente, o que acarreta o uso de meios práticos e mais acessíveis, como a utilização de enlatados e lanches, presentes, frequentemente, na rotina alimentar de universitários.

Em relação à ocorrência do estresse, estudos mostram que os universitários têm demonstrado vários agentes estressantes, devido às fortes preocupações com apresentação e entrega de trabalhos acadêmicos, período de provas e final de semestre (Coelho \& Santos, 2006). Esses dados negativos sobre o controle do estresse podem ser decorrentes de aspectos negligenciados pelos universitários do presente estudo, por exemplo, no item $\mathrm{F}$, aquele que se refere à atividade física diária, os universitários mostraram escores negativos. Segundo a OMS (2006), a prática efetiva da atividade física é uma grande aliada no combate ao estresse porque traz melhoras nos níveis gerais, evita o desgaste emocional e proporciona bem-estar.

Dentro dessa perspectiva sistemática sobre atividade física, saúde e qualidade de vida, buscou-se saber, ainda, juntos aos universitários que compuseram a amostra do estudo, sobre o nível da atividade física habitual. Isto porque, em um estudo desenvolvido por Perioto (2010) com estudantes, usando essas mesmas características metodológicas, encontraram-se índices de inatividade física maiores que os do presente estudo. Os resultados inversos podem ser decorrentes dos pensamentos mais críticos dos universitários de Educação Física sobre a importância de um programa de vida ativa, tanto no lazer quanto na vida diária.

Na estratificação dos resultados das atividades físicas habituais dos universitários dos cursos de licenciatura e bacharelado em Educação Física, conseguimos identificar, nos dados da pesquisa, a informação de que nenhum universitário de licenciatura tem associação inversa entre níveis de atividade física e a possibilidade incidência de 
patologias que tenham relação com ausência de atividades físicas habituais; já quanto aos alunos do bacharelado, 28,9\% são inativos ou pouco ativos, caracterizados como sedentários. Nesse sentido, Nahas (2010) considera a atividade física um meio possível para desencadear um estilo de vida mais saudável e de combate ao sedentarismo.

\section{Conclusão}

Diante dos resultados, este estudo foi especialmente útil, pois sugere que os estilo de vida dos universitários dos cursos de licenciatura e bacharelado da amostra estudada apresentam pelo menos um escore negativo em cada um dos cinco componentes estudados. Diante disso, ficou observado que os componentes nutrição e estresse apresentam os maiores índices de escores negativos, tanto para o curso de licenciatura quanto para o de bacharelado.

Torna-se necessário relatar que os dados demonstram uma prevalência negativa nos aspectos fundamentais para a saúde geral. Diante disso, é imprescindível que os sujeitos dos estudos identificados com escores negativos se conscientizem de que precisam melhorar seus hábitos alimentares e controlar seus estresses diários, relaxar durante seus maiores momentos de tensão acadêmica, pois estes impasses poderão acarretar problemas relacionados à saúde e baixos níveis de qualidade de vida.

Quanto à análise do nível de atividade física habitual, os dados apresentam-se como bons indicadores do nível de vida fisicamente ativo da amostra estudada. No entanto, de modo específico, uma parcela dos indivíduos do curso de bacharelado em Educação Física apresentou índice indesejável, sendo caracterizada como indivíduos abaixo dos níveis considerados ideias para a saúde física.

Nesta perspectiva, nota-se que os resultados obtidos em relação a atividade física habitual demonstram uma prevalência de inatividade física. Assim, sendo esse um problema de saúde pública, torna-se, portanto, indispensável que haja uma reflexão direta sobre esses aspectos apontados, já que esses resultados podem contribuir para incrementar a atividade física diária, proporcionando aos universitários os caminhos para a adoção de um estilo de vida ativo.

\section{Referências}

Barboza, C. F., Pontes, L. M., Feitosa, K.V.J., Nunes, J.R.A. \& Sena, J.E.A (2011). Estilo de vida e prevalência de excesso de peso em universitários de educação física de instituição particular de ensino superior de João Pessoa. Coleção Pesquisa em Educação Física, 10(1), 89-96. Recuperado de http://www.editorafontoura.com.br/periodico/vol-10/Vol10n1-2011/Vol10n1-2011-pag-89a96/Vol10n1-2011-pag89a96.pdf

Bauman, A.E., Reis, R.S., Sallis, J.F., Wells, J.C., Loos, R.J. \& Martin B.W. (2012). Lancet Physical Activity Series Working Group. Correlates of physical activity: why are some people physically active and others not? Lancet, 380, 258-271. Recuperado de http://www.thelancet.com/pdfs/journals/lancet/PIIS0140-6736(12)607351.pdf

Barros, M.V.G. \& Nahas, M. V. (2001). Comportamentos de risco, auto-avaliação do nível de saúde e percepção de estresse entre trabalhadores da indústria. Revista de Saúde Pública, 35(6), 554-563.

Both, J., Nascimento, J.V., Sonoo, C.N. \& Borgato, A.F.(2010). Condições de vida do trabalhador docente: Associação entre estilo de vida e qualidade de vida no trabalho de professores de Educação Física. Revista Motricidade, 6(3), 39-51. Recuperado de http://www.revistamotricidade.com/arquivo/2010 vol6 n3/v6n3a06.pdf

Coelho, C. W. \& Santos, J.F.S. (2006). Perfil do estilo de vida relacionado à saúde dos calouros de um centro de ciências tecnológicas. Revista Educación Fisica y Deportes, 11(97), 1-7. Recuperado de http://www.efdeportes.com/efd97/saude.htm 
Cruz, A. A. M. (2006). Caracterização do Perfil de Atividade Física e sua Relação com os Indicadores de Saúde em Indivíduos de Etnia Japonesa Residentes na Região Metropolitana de Porto Alegre - RS. Porto Alegre, 123fls.Tese (Curso de Doutorado em Medicina e Ciência da Saúde). Pontifícia Universidade Católica do Rio Grande do Sul. Porto Alegre. Recuperado de http://repositorio.pucrs.br/dspace/handle/10923/4322

Hallal, P.C., Andersen, L.B., Bull, F.C., Guthold, R., Haskell W. \& Ekelund, U. (2012). Lancet Physical Activity Series Working Group. Global physical activity levels: surveillance progress, pitfalls, and prospects. Lancet, 380, 247-257. Recuperado de http://www.thelancet.com/journals/lancet/article/PIIS0140-6736(12)60646-1/fulltext

Nahas, M. V., Barros, M. V. G., Francalacci, V. L. (2000). O pentáculo do bem-estar: base conceitual para avaliação do estilo de vida de indivíduos e grupos. Revista Brasileira de Atividade Física \& Saúde, 5(2), 48-59. Recuperado de https://periodicos.ufpel.edu.br/ojs2/index.php/RBAFS/article/view/1002

Nahas, M. V. (2010). Atividade física, saúde e qualidade de vida: conceitos e sugestões para um estilo de vida ativo. - $5^{\mathrm{a}}$ Ed. Ver. E atual.-Londrina: Midiograf. Recuperado de http://www.scielo.br/scielo.php? $\underline{\text { script }=\text { sci } \_ \text {arttext } \& \text { pid }=\text { S0101-32892012000200018 }}$

Nogueira A.P, Rossato, M., Soares, A.A., Bezerra, E.S. \& Moraes M.S. (2015). O estilo de vida dos universitários da Faculdade de Educação Física e Fisioterapia da UFAM. Recuperado de https://www.researchgate.net/publication/28089872 O estilo de vida dos universitarios da Faculdade de Edu cacao Fisica e Fisioterapia da UFAM.

Organização Mundial de Saúde - OMS (2006). Atividade física e saúde na Europa: Evidências para a acção. Centro de Investigação em Actividade Física, Saúde e Lazer. Porto. Recuperado de https://ciafel.fade.up.pt/files download/actividade fisica e saude na europa.pdf

Pratt, M., Sarmiento, O.L., Montes, F., Ogilvie, D., Marcus, B.H., Perez, L.G. \& Brownson R. (2012). The implications of megatrends in information and communication technology and transportation for changes in global physical activity. Lancet, 380, 282-293. Recuperado de http://www.thelancet.com/journals/lancet/article/PIIS0140-6736(12)60736-3/fulltext

Perioto, J. F. M. (2010). Relação entre nível de atividade física e estado nutricional em escolares. Trabalho de Conclusão de Curso (Graduação em Educação Física) - Universidade Estadual de Maringá - UEM.

Pereira, A., Motta, E., Vaz, A., Pinto, C., Benardino, O., et. al. (2006). Sucesso e desenvolvimento psicológico no ensino superior: estratégias de intervenção. Revista Análise Psicológica, 24, 51-59. Recuperado de http://www.scielo.mec.pt/scielo.php?script=sci arttext\&pid=S0870-82312006000100006

Santos, J.F.S. \& Alves, V.S. (2009). Perfil do estilo de vida relacionado à saúde de acadêmicos da Unicentro, Campus, Irati, PR. Revista digital, 13(129). Recuperado de http://www.efdeportes.com/efd129/perfil-do-estilo-devida-relacionado-a-saude-dos-academicos.htm

Silva, D. A. S., Pereira, I.M.M., Almeida, M. B., Silva, R.J.S. \& Oliveria, A.C.C. (2012). Estilo de vida de acadêmicos de educação física de uma universidade pública do estado de Sergipe, Brasil. Revista Brasileira de Ciências do Esporte, 34(1), 53-67. Recuperado de http://dx.doi.org/10.1590/S0101-32892012000100005 